\title{
Monte Carlo simulation of a CPO beam line: modelling the nuclear interactions
}

\author{
A. Stankovskiy ${ }^{1, a}$, S. Kerhoas-Cavata ${ }^{1}$, R. Ferrand ${ }^{2}$, and C. Nauraye ${ }^{2}$ \\ ${ }^{1}$ CEA/DSM/DAPNIA/SPhN, 91191 Gif-sur-Yvette Cedex, France \\ 2 Centre Protonthérapie d'Orsay, Bât. 101 du Centre Universitaire d'Orsay, BP. 65, 91402 Orsay Cedex, France
}

\begin{abstract}
The Proton Therapy Center in Orsay (CPO) and CEA/DAPNIA launched the joint project on Monte Carlo modeling of a $\mathrm{CPO}$ beam line with the aim to achieve a prediction of dose distribution in all the calibration configurations (depth and the shape of the tumor) better than $2 \%$. The calculation module is intended to be used for the absolute dosimetry of the clinical beam (patient Quality Assurance - QA), and in a second stage for predicting the dose distribution on a voxelized phantom constructed from the Computer Tomography (CT) patient's data. The MCNPX code was used as a basic Monte Carlo simulation tool in this study. All the elements of a CPO beam line were modelled with sub-millimetric precision. To speed up the calculations of modulated dose profiles in water phantom, a routine simulating rotation of modulator wheel was created to save phase space of particle tracks crossing the modulator. The modeling of nuclear interactions, which contribute noticeably into the total absorbed dose in water phantom at the prescribed proton energy (average raw beam energy $201 \mathrm{MeV}$ ), was improved by means of creation and implementation of the two new evaluated proton-induced nuclear data files up to $200 \mathrm{MeV}$ for ${ }^{1} \mathrm{H}$ and ${ }^{16} \mathrm{O}$. Thus, the simulations of 3D dose profiles in water show excellent agreement with measured data allowing to move forward to the absolute dose calibrations.
\end{abstract}

\section{Introduction}

The Monte Carlo (MC) modeling of radiation transport might be the effective way to substitute the conventional tools for calculation the dose distribution inside a patient used for treatment planning in proton therapy. The latter usually is based on various parameterizations of measured proton dose distributions (so-called pencil-beam or broad-beam techniques) while the Monte Carlo simulation provides the accurate description of the almost all possible interactions of particles with matter. The disadvantage is still huge computational time making it difficult implementing in daily clinical routine.

The Proton Therapy Center in Orsay (CPO) and CEA/DAPNIA launched the joint project on Monte Carlo modeling of a CPO beam line with the aim to achieve the targeted accuracy prediction of $3 \mathrm{D}$ dose distribution in all the calibration configurations (depth and the shape of the tumour) better than 2\% and 1-2 $\mathrm{mm}$ in range. The calculation module is intended to be used for the absolute dosimetry of the clinical beam (patient QA), and in a second stage for predicting the dose distribution on a voxelized phantom constructed from the CT patient's data. Along with this ultimate goal, the Monte Carlo simulation tool could be helpful in better understanding of fundamental physical processes taking place in the beam delivery system and, probably, better configuration of a beam line.

The required predictive accuracy of a Monte Carlo tool necessitates to carefully describe all physical processes within it. Despite the main mechanism for protons to loose their energy along flight path is atomic interaction, they may undergo nuclear interactions, and produce secondary particles which contribute to the dose. This contribution rises with primary proton energy. Moreover, the careful modeling of secondary neutrons produced from interactions of protons with

${ }^{a}$ Present author, e-mail: Alexey.Stankovskiy@cea.fr matter is especially important in the shielding problems. This contribution describes the realization of nuclear interactions within Monte Carlo modeling of a CPO beam line.

\section{Methods}

The general purpose Monte Carlo transport code MCNPX 2.5.0 [1] was chosen as the tool to perform simulations. The brief description and basic features of MCNPX as applied to the simulation of proton therapy could be found in $[2,3]$.

The CPO beam line simulated by Monte Carlo is schematically described in figure 1.

This line uses passive scattering technique to provide a flat lateral dose profile and distribute the dose homogeneously in depth to create a spread-out Bragg peak (SOBP). The raw beam of nearly Gaussian shape in space and energy (mean energy $201 \mathrm{MeV}$ with the energy spread $0.5 \%$ that corresponds to full-width-at-half-maximum $1.005 \mathrm{MeV}$; angular spread $\Delta \theta=4.4 \mathrm{mrad}$; pulses of $20 \mu \mathrm{s}$, produced at a frequency of $448 \mathrm{~Hz}$ ), coming from the accelerator tube, first faces the rotating wheel which modulates the proton energy spectrum. Between the accelerator tube vacuum window and the modulator, two transmission ion chambers are situated to monitor beam parameters. After being cut by the first collimator with diameter $3 \mathrm{~cm}$, the beam interacts with the range shifter (so-called "binary filter") that consists of light (lexan, density $1.2 \mathrm{~g} / \mathrm{cm}^{3}$ ) and heavy material (lead) layers of various thicknesses. The patient-dependent range and flatness of the dose distribution are formed by the proper combination of these range shifting and pre-scattering layers.

Another element of the double-scattering system, so-called "scatterer", consists of the set of thin lead foils put together as it is shown in figure 1 . Then beam enters the treatment room through a hole in the concrete shielding separating the treatment room from the accelerator beam line. After 


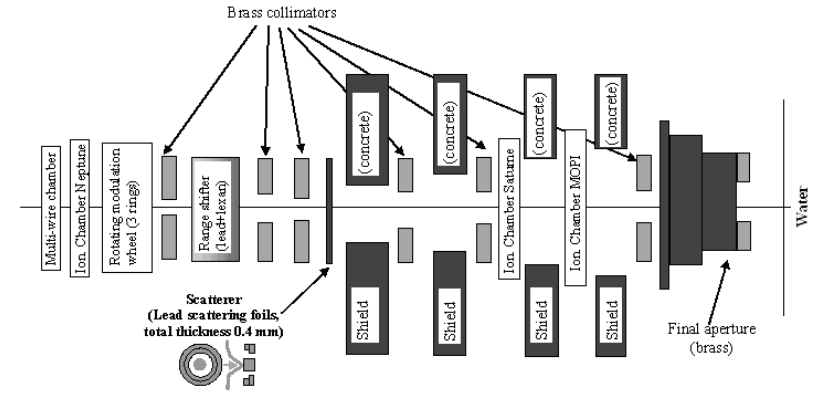

Fig. 1. Composition of beam line (not to scale). Beam is entering from the left. The distance from the accelerator tube vacuum window (not shown) to water tank is about $7 \mathrm{~m}$.

passing through a number of collimators and ion chambers, the beam is finally collimated and protons deposit their energy in water. In the clinical case, the patient-dependent collimators and compensators to cover the tumour shape are attached to the final aperture. All the elements of a CPO beam line were modelled according to the technical data provided by manufacturer.

\subsection{Modulated beam}

The simulation of modulated beam, in general, represents certain difficulties when using MCNPX since the code does not treat time-dependent geometry changes. However, for the modeling of CPO beam line geometry set-up, it was easily avoided. The idea behind that was to create phase space files just after modulator. The aluminium modulator wheel consists of three "rings" with sectors of various thicknesses. It was described within sub-millimeters in terms of MCNPX combinatorial geometry, and the routine realizing "quasi-timedependent" simulation was created to save information on particles crossing modulator after each rotation step. This routine performs automatic transformation of the modulator geometry according to the value of rotation angle, launches the MCNPX simulation in single processor mode (parallel calculation mode was found to be ineffective since time to send/receive subtasks from master to slave processors is comparable with typical simulation time on single processor for each angular step) and adds particle track information to surface-source write (SSW) file. It was found that to have smooth particle distributions (for each particle type the energy, position, direction and momentum were scored) an angular rotation step of 2 degrees is enough with a number of primary proton tracks simulated per angular step equal to 100,000. Three surface-source read (SSR) files containing phase space for each modulation ring were created.

The use of these three distributions as new particle sources allowed to reduce significantly (up to a factor of 100) the computation time. Moreover, the actual number of particle tracks (limited by the available disk storage capacity, at present about 9 millions tracks are stored in each file) might be increased in subsequent simulation to ensure the better quality of results. Each starting particle track is repeated with new random number seed. The recycling factor is ranged from 2 (for unmodulated depth profiles) to several hundreds (for lateral profiles).
Typical computing time to obtain SOBP of the quality better than $2 \%$ for dose and $1 \mathrm{~mm}$ for range and modulation width is about 15 min to simulate $10^{7}$ particle histories originated from the phase space right after modulator. The cluster of nine $2 \mathrm{GHz}$ processors running under Linux was used to perform calculations for this study. This seems to be acceptable from the viewpoint of further integration of Monte Carlo dose calculation into the treatment planning.

\section{Results and discussion}

Typically the secondary particles, originated from nuclear non-elastic interactions with materials along the beam line, contribute insignificantly to the total dose delivered to water [4]. The exception is secondary protons whose contribution might be as high as $10-15 \%$ to the energy deposited close to single Bragg peak. In addition, in the case of SOBP, they influence the flatness and may undergo other nuclear interactions. Other particles generated in nuclear interactions (deuterons, tritons, ${ }^{3} \mathrm{He}$ and alphas) are contributing less than $2 \%$. Neutrons, electrons and photons deposit their energy far outside the irradiated volume while their contribution to dose to water is approximately $0.1 \%$.

Taking into account the primary goal to fast and correctly reproduce with MCNPX the $3 \mathrm{D}$ dose distribution in the water phantom, the neutrons, photons and electrons were removed from the list of transported particles. Applying variance reduction techniques speeds up to 15 times the calculation of dose profiles. However, such problems as the study of the dose delivered by neutrons (or photons and electrons) outside the irradiated volume, as well as estimation of output factors for beam calibration, will obviously require tracking all particles.

In general it is of great importance to carefully account for nuclear interactions (both elastic and non-elastic) which take place close to and inside in the irradiated volume, i.e. water tank in our case. MCNPX has two options for treating nuclear interactions: to invoke various physics models (up to 8 combinations of intranuclear cascade, preequilibrium and evaporation models) or to sample them according to nuclear data tables read in from corresponding files. In case the data table for particular isotope is absent or the energy range of data does not span the energies characteristic to the problem, the code uses model calculations. Strictly speaking, especially for the energies characteristic to proton therapy (up to $\sim 200$ $-250 \mathrm{MeV}$ ) the data files provide more precise description of nuclear interactions than the inherent models in MCNPX for two reasons: the proton induced data are evaluated from the external model calculations and experimental data, and, secondly, the quality of the preequilibrium and evaporation models in MCNPX which dominate at these energies remains questionable.

The dose profiles shown in figure 2 were calculated with proton-induced cross section data from LA150H library [5] (solid curve) and physics models for nuclear interactions inherent in MCNPX (dash-dotted curve). Additional tests proved the fact that contribution of nuclear interactions occurred outside the water tank into the total dose is vanishingly small and the difference between two cases is mostly due to the difference in cross sections read in from data files and 


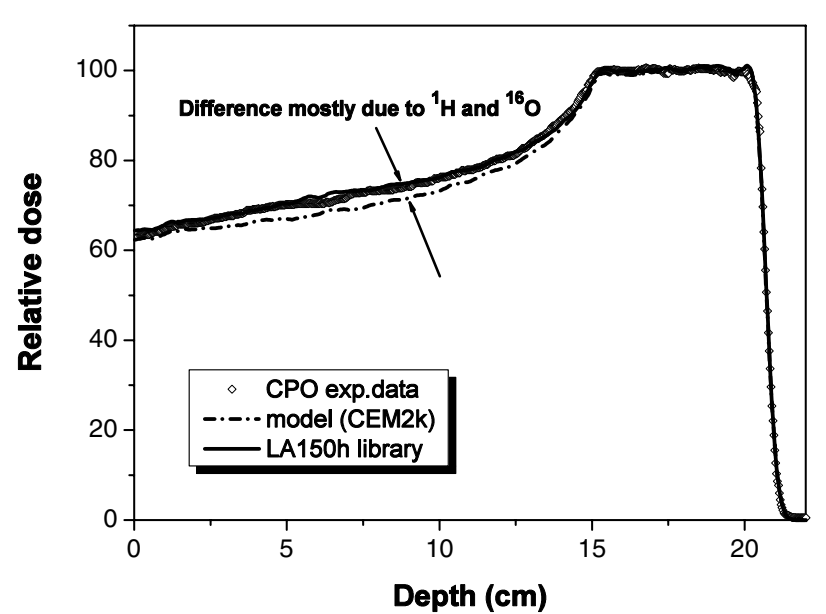

Fig. 2. Depth dose distribution for modulated beam calculated with model option, LA150H library, and new data files.

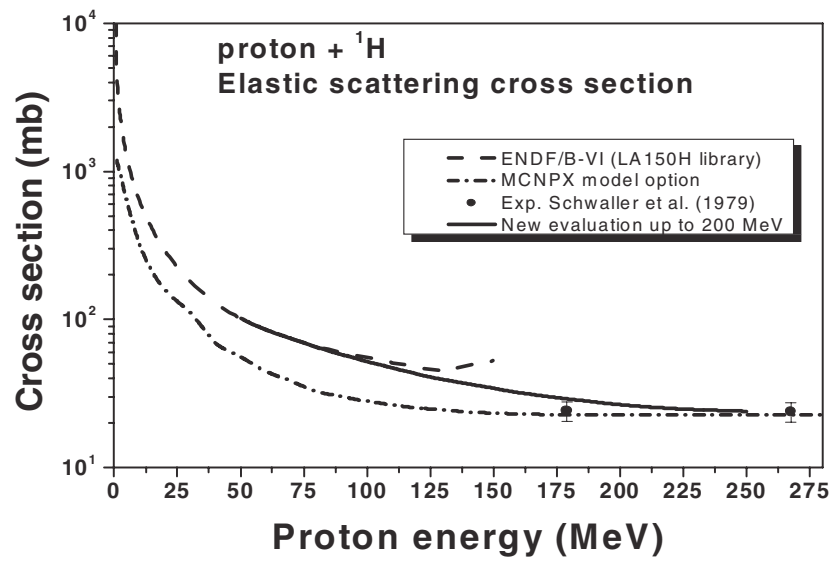

Fig. 3. Elastic scattering cross sections of protons by nuclei of ${ }^{1} \mathrm{H}$. Experimental data are taken from the EXFOR library [http://www.nndc.bnl.gov/exfor3/exfor00.htm].

modelled inside MCNPX for two most important isotopes hydrogen-1 and oxygen-16 which almost compose the water.

However, the data files, in general, have upper energy limit of $150 \mathrm{MeV}$ which is not enough to fully cover the problem. This resulted in the two new evaluated data files for $\mathrm{p}+{ }^{1} \mathrm{H}$ and $\mathrm{p}+{ }^{16} \mathrm{O}$ up to $200 \mathrm{MeV}$ briefly described below.

\subsection{Protons $+{ }^{1} \mathrm{H}$}

Figure 3 shows the comparison of elastic scattering cross sections which are contained in the proton induced data file for H-1 from LA150H library distributed with MCNPX and those which are used by MCNPX models. As it might be seen, the cross section used by MCNPX models is significantly less than the one contained in ENDF/B-VI (LA150H) file [5].

A new evaluation up to $200 \mathrm{MeV}$ on the basis of available experimental data has been performed. The new proton elastic scattering cross section seems to be more accurate. The data were written in ENDF-6 format and subsequently processed by the NJOY99 code [6] to generate data in ACE format acceptable by MCNPX.
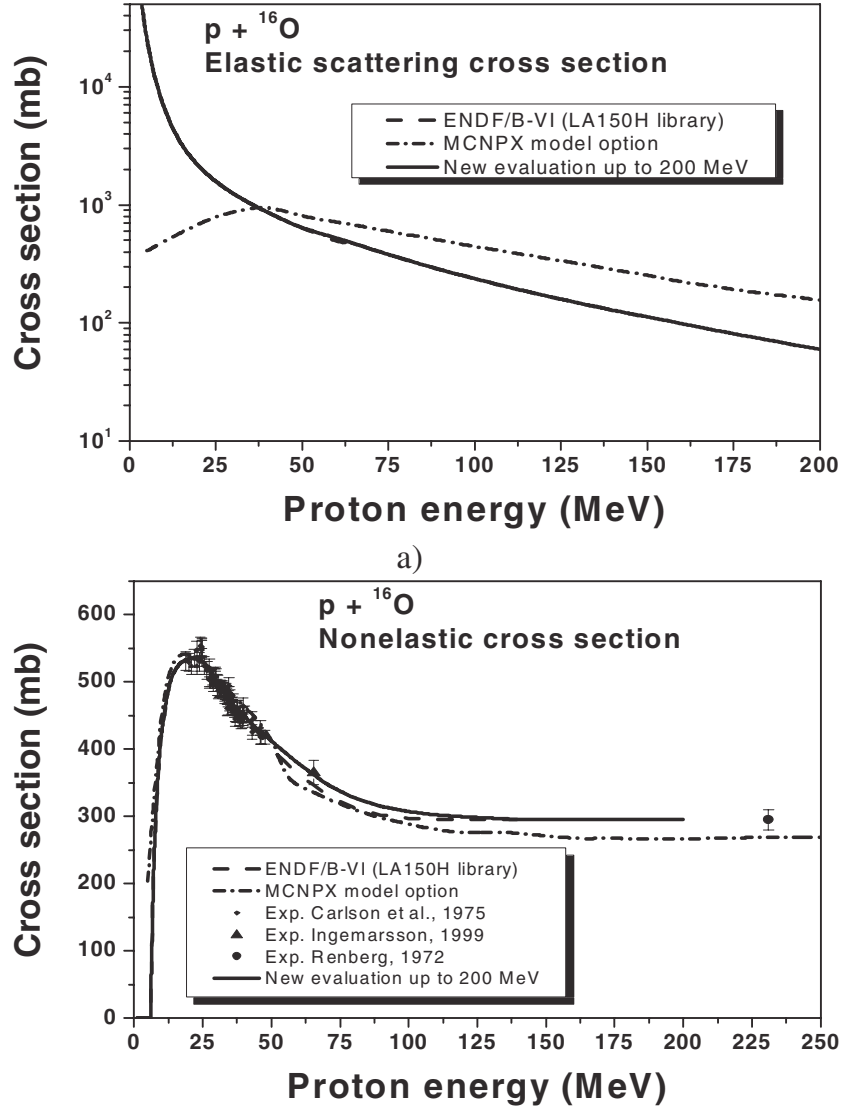

b)

Fig. 4. Elastic scattering (a) and total non-elastic reaction (b) cross sections of protons by nuclei of ${ }^{16} \mathrm{O}$. Experimental data are taken from EXFR [http://www.nndc.bnl.gov/exfor3/exfor00.htm].

\subsection{Protons $+{ }^{16} \mathrm{O}$}

Elastic scattering cross section and total non-elastic reaction cross sections are shown in figure $4(a, b)$.

Elastic scattering cross section up to $150 \mathrm{MeV}$ was taken from ENDF/B-VI file [5] with small adjustment between 40 and $70 \mathrm{MeV}$; the values between 150 and $200 \mathrm{MeV}$ were calculated with the help of the TALYS [7] code and joined to ENDF/B-VI data.

Non-elastic reaction cross sections were evaluated on the basis of TALYS calculations and experimental data taken from EXFOR compilation.

The new evaluated file contains also the production cross sections and emission spectra for neutrons, protons, deuterons, tritons, He-3, alpha particles and $\gamma$-rays. Below $150 \mathrm{MeV}$, the data were taken from the ENDF/B-VI file [5]. Above $150 \mathrm{MeV}$, the evaluation was performed with the help of the ALICE/ASH code [8]. The description of nuclear level density was obtained on the basis of the generalized superfluid model [9]. The pre-equilibrium alpha-particle emission spectra were calculated with the help of the coalescence pickup model [10] combined with the knock-out model [11]. The multiple pre-equilibrium emission was taken into account. The deuteron, triton and ${ }^{3} \mathrm{He}$ precompound spectra were calculated with the help of the exciton pick-up model from [12]. The 
Table 1. Values of factors $\mathrm{H}$ and $\mathrm{D}$ for different beam line configurations.

\begin{tabular}{ccccccc}
\hline \multirow{2}{*}{$\begin{array}{c}\text { Range } \\
(\mathrm{cm})\end{array}$} & $\mathrm{H}$ & $\mathrm{LA150H}$ & \multicolumn{2}{c}{ Model (CEM2k) } & \multicolumn{2}{c}{ New files } \\
\hline 20.4 & 0.923 & $1.50 \times 10^{-2}$ & 2.140 & $4.11 \times 10^{-2}$ & 0.901 & $1.35 \times 10^{-2}$ \\
19.9 & 1.003 & $3.20 \times 10^{-3}$ & 1.734 & $2.20 \times 10^{-2}$ & 0.855 & $5.45 \times 10^{-4}$ \\
16.3 & 1.275 & $3.19 \times 10^{-3}$ & 1.643 & $1.99 \times 10^{-2}$ & 1.216 & $4.05 \times 10^{-4}$ \\
10.3 & 1.213 & $3.05 \times 10^{-3}$ & 2.701 & $2.49 \times 10^{-2}$ & 1.167 & $1.03 \times 10^{-3}$ \\
\hline
\end{tabular}

phenomenological approach from [13] was used to obtain the contribution of the direct mechanism to the deuteron emission.

The extended description of the methods of data evaluation is presented in [14].

The analysis with the help of coincidence criteria for several configuration options giving different range of protons in water manifests that the use of new evaluated data files gives more precise depth dose distributions. This is vividly seen from table 1 . The following criteria were used:

$$
H=\frac{1}{N} \sqrt{\sum_{i=1}^{N}\left(d_{i}^{\exp }-d_{i}^{c a l c}\right)^{2}}
$$

and

$$
D=\frac{1}{N} \sum_{i=1}^{N}\left|\frac{d_{i}^{\exp }-d_{i}^{c a l c}}{d_{i}^{\exp }}\right|
$$

where $d_{i}^{\text {exp }}$ and $d_{i}^{\text {calc }}$ stand for experimental and calculated doses at $i$-th depth in water.

In the same time, due to the large underestimation of proton elastic scattering cross section on hydrogen below $150 \mathrm{MeV}$, the MCNPX model option significantly underestimates the shape of the curve (blue curve in fig. 5). In case of SOBP the difference between model calculations and calculations with the use of evaluated data files is more pronounced, than in case of single non-modulated Bragg curve, due to accumulation of this discrepancy when summing up the doses deposited by protons at each angular position of modulator wheel.

There was found no dependence of distal fall-off edge of the SOBP and lateral dose profile from the model/data choice. The SOBP was simulated with accuracy below the experimental uncertainties, i.e., within prescribed value of $1 \mathrm{~mm}$.

\section{Conclusions}

Monte Carlo simulation for CPO treatment head geometry has been realized using MCNPX 2.5.0 code. The validation performed with reference data reveals good agreement within $1-2 \mathrm{~mm}$ in range, modulation width, distal and lateral penumbra for all sets of data (more than 40 depth and lateral dose distributions for modulated and unmodulated beam were simulated). The novel approach to simulate time-dependent geometry with MCNPX was proposed. The use of new evaluated proton-induced nuclear data files up to $200 \mathrm{MeV}$ for hydrogen-1 and oxygen-16 allowed to improve the predictive power of MCNPX dose calculations.

\section{References}

1. D.B. Pelowitz (ed.), MCNPX User's Manual, version 2.5.0 LACP-05-0369, 2005.

2. J. Hérault, N. Iborra, B. Serrano, P. Chauvel, Med. Phys. 32, 910 (2005).

3. W. Newhauser, N. Koch, S. Hummel, M. Ziegler, U. Titt, Phys. Med. Biol. 50, 5229 (2005).

4. H. Paganetti, Phys. Med. Biol. 47, 747 (2002).

5. M.B. Chadwick, P.G. Young, R.E. MacFarlane et al., Los Alamos National Laboratory report LA-UR-99-1222, 1999.

6. R.E. MacFarlane, NJOY99-Code system for producing pointwise and multigroup neutron and photon cross sections from ENDF/B Data, RSICC PSR-480, 2000.

7. A.J. Koning, S. Hilaire, M.C. Duijvestijn, in Proceedings of the International Conference on Nuclear Data for Science and Technology-ND2004, Santa Fe, USA (2004), p. 1154.

8. C.H.M. Broeders, A.Yu. Konobeyev, Yu.A. Korovin, V.P. Lunev, M. Blann, Report FZKA 7183, 2006.

9. A.V. Ignatyuk, IAEA-TECDOC-1034, Chapter 5 (1998).

10. A. Iwamoto, K. Harada, Phys. Rev. C 26, 1821 (1982).

11. A.Yu. Konobeyev, V.P. Lunev, Yu.N. Shubin, Acta Physica Slovaca 45, 705 (1995).

12. N.Sato, A. Iwamoto, K. Harada, Phys. Rev. C 28, 527 (1983).

13. A.Yu. Konobeyev, Yu.A. Korovin, Kerntechnik 61, 45 (1996).

14. Yu.A. Korovin, A.Yu. Konobeyev, G.B. Pilnov, A.Yu. Stankovskiy, Nucl. Instrum. Meth. Phys. Res. A 562, 721 (2006). 